Bundesgesundheitsbl 2013 · 56:1432-1432 DOI 10.1007/s00103-013-1848-5

Online publiziert: 25. September 2013

(c) Springer-Verlag Berlin Heidelberg 2013
U. Maschewsky-Schneider ${ }^{1} \cdot$ M. Goecke ${ }^{2} \cdot$ U. Hölscher ${ }^{3} \cdot$ P. Kolip ${ }^{4} \cdot$ A. Kuhn ${ }^{5}$. D. Sewöster ${ }^{6} \cdot$ H. Zeeb ${ }^{7}$

${ }^{1}$ Berlin School of Public Health, Charité - Universitätsmedizin Berlin

${ }^{2}$ Bundeszentrale für gesundheitliche Aufklärung, Köln

${ }^{3}$ Zentrum für Medizintechnik und Ergonomie, Fachhochschule Münster

${ }^{4}$ Fakultät für Gesundheitswissenschaften, Universität Bielefeld

${ }^{5}$ Geschäftsstelle gesundheitsziele.de, Gesellschaft für

Versicherungswissenschaft und Gestaltung (GVG) e. V., Köln

${ }^{6}$ Deutsche Rentenversicherung Bund, Berlin

${ }^{7}$ BIPS, Universität Bremen

\section{Erratum zu: Gesundheitsziele.de. Die Auswahl eines neuen Gesundheitsziels für Deutschland}

Im oben genannten Beitrag wurde der Nachname der Autorin M. Goecke leider falsch angegeben. Wir bitten, die korrekte Nennung zu beachten und den Fehler zu entschuldigen.

Die Redaktion

\section{Korrespondenzadresse}

Prof. Dr. U. Maschewsky-Schneider

Berlin School of Public Health,

Charité - Universitätsmedizin Berlin

Gebäude A, Aufgang 9, 4. OG,

Oudenarder Str. 16, 13347 Berlin

ulrike.maschewsky-schneider@charite.de 